\title{
Increase of the therapeutic effect by treating nasopharyngeal tumor with combination of HER-2 siRNA and paclitaxel
}

\author{
YOSHIYUKI HATTORI, MOTOKI HAKOSHIMA, KIMIKO KOGA and YOSHIE MAITANI \\ Institute of Medicinal Chemistry, Hoshi University, Shinagawa-ku, Tokyo 142-8501, Japan
}

Received November 26, 2009; Accepted January 22, 2010

DOI: 10.3892/ijo_00000585

\begin{abstract}
Therapeutic agents targeting HER-2/neu have been intensively addressed over the past decades. Previously, we reported that HER-2 synthetic small interfering RNA (HER-2 siRNA) could suppress the growth of human nasopharyngeal KB tumor xenografts by intratumoral injection with lipidbased nanoparticles; however, complete regression of the tumor was not achieved. In this study, we investigated antitumor activity by RNA interference in combination with paclitaxel (PTX) for KB cells using HER-2 siRNA and HER-2 short hairpin RNA-expressing plasmid DNA (HER-2 shRNA pDNA). Suppression of HER-2 expression by siRNA or shRNA pDNA caused significant reduction of proliferation by inducing apoptosis and enhancing the sensitivity for PTX in HER-2 positive KB cells. Interestingly, an HER-2 antibody trastuzumab could not increase the antitumor effect by PTX in KB xenografts. Combination therapy by intratumoral injection of HER-2 siRNA or HER-2 shRNA pDNA with PTX significantly inhibited the tumor growth of xenografts compared with each therapy used individually. In particular, HER-2 shRNA pDNA plus PTX largely extended the mean survival days compared with HER-2 siRNA plus PTX. Collectively, these findings suggest that HER-2 shRNA-based combined therapy with PTX could be a novel strategy to inhibit the progression of HER-2-positive cancer.
\end{abstract}

\section{Introduction}

The HER-2 proto-oncogene (c-erbB-2/neu) belongs to the epidermal growth factor (EGF) receptor family and has been implicated in malignant transformation (1). HER-2 is thought to play an important role in tumorigenesis, DNA repair, drug resistance, and metastasis. HER-2 gene expression has been found to be amplified and/or overexpressed in breast (2), ovarian (3), lung (4), prostate (5), thyroid (6) and pancreatic cancers (7). HER-2 overexpression induced the down-regulation of p53 protein through the PI3 K/AKT

Correspondence to: Dr Yoshiyuki Hattori, Institute of Medicinal Chemistry, Hoshi University, Shinagawa-ku, Tokyo 142-8501, Japan E-mail: yhattori@hoshi.ac.jp

Key words: synthetic siRNA, cationic nanoparticles, nasopharygeal tumor, HER-2 pathway (8), leading to increased cell proliferation and decreased sensitivity to chemotherapeutic drugs (9); therefore, HER-2 has become an important target for cancer therapy.

Interference with HER-2 mRNA translation could be more effective than blockade of the already expressed HER-2 on the cell surface by HER-2 antibody. Specific down-regulation of HER-2 expression in tumors by ribozyme (10-12), antisense DNA (13-15), small interfering synthetic siRNA nucleotide (siRNA) $(16,17)$, plasmid DNA (pDNA) encoding a short hairpin RNA (shRNA pDNA) (18) and antisense RNA/sense DNA hybrid duplexes (19) have been reported. Successful down-regulation of HER-2 will require sustained and high transfection of the therapeutic gene. RNA interference (RNAi) is a post-transcriptional mechanism of gene silencing mediated by cleavage of target RNA. RNAi has potential not only as a tool in biological analysis, but also as an evolutional drug for cancer gene therapy. In RNAi technology, two delivery systems were considered; direct delivery of siRNA, and introduction of shRNA pDNA that will be enzymatically degraded into siRNA. Synthetic siRNAs, which are 21-28 bp small doublestranded RNA, are substrates for the RNA-induced silencing complex.

In a previous study, we reported that HER-2 synthetic small interfering (HER-2) siRNA could suppress the growth of human nasopharyngeal KB tumor xenografts by intratumoral injection with lipid-based nanoparticles (NP) (20); however, complete regression of the tumor was not observed. Recently, it has been reported that combination therapy with HER-2 antibody trastuzumab and paclitaxel (PTX) is useful for the clinical treatment of breast cancer (21-23). Thus, it was expected that combination therapy with HER-2 siRNA and chemotherapy would be effective; however, it has not been reported in in vivo models. Therefore, in the present study, we evaluated the potential of HER-2 siRNA and HER-2 short hairpin RNA-expressing plasmid DNA (HER-2 shRNA pDNA) using NP delivery to increase the therapeutic efficacy of PTX in HER-2-positive KB cells and tumor xenografts.

\section{Materials and methods}

Synthetic siRNA. The stealth RNA interference duplextargeting nucleotides of HER-2 mRNA (HER-2 siRNA) and stealth RNAi Negative Control kit with Medium GC as a control for HER-2 siRNA (Cont siRNA) were synthesized by Invitrogen (Carlsbad, CA, USA). The sequences of HER-2 siRNA were as follows: HER-2 sense, 5'-AAACGUGUCUG 
UGUUGUAGGUGACC-3'; HER-2 antisense, 5'-GGUCAC CUACAACACAGACACGUUU-3'.

shRNA expressing plasmid DNA. HER-2 shRNA pDNA encoding 21 mer shRNA against HER-2 with a hairpin-loop under the control of the U6 promoter (HuSH 19-21 mer shRNA construct against ERBB2) was obtained from OriGene Technologies, Inc. (MD, USA). The targeted sequences of HER-2 shRNA pDNA was as follows: HER-2 sense, 5'-AGTGAGCACCATGGAGCTGGC-3'; HER-2 antisense, 5'-GCCAGCTCCATGGTGCTCACT-3'. The pRS-shGFP (29) non-effective plasmid (Cont shRNA pDNA, OriGene Technologies, Inc.) was used as a negative control. A protein-free preparation of these plasmids was purified following alkaline lysis using the EndoFree Plasmid Max kit (Qiagen, Hilden, Germany).

Cell culture. Human nasopharyngeal tumor KB cells were supplied by the Cell Resource Center for Biomedical Research, Institute of Development, Aging and Cancer, Tohoku University (Miyagi, Japan). Human cervix epithelial adenocarcinoma HeLa cells were obtained from the European Collection of Cell Culture (Wiltshire, UK). Human lung carcinoma A549 cells were a gift from Oncotherapy Science (Tokyo, Japan). KB and A549 cells were grown in RPMI-1640 medium (Invitrogen) and HeLa cells in Eagle's Minimum Essential Medium (Invitrogen), supplemented with 10\% heatinactivated fetal bovine serum (FBS, Invitrogen) and kanamycin $(100 \mu \mathrm{g} / \mathrm{ml})$ at $37^{\circ} \mathrm{C}$ in a $5 \% \mathrm{CO}_{2}$ humidified atmosphere.

In vitro and in vivo transfection. For in vitro and in vivo transfections of siRNA and shRNA pDNA, we used lipidbased nanoparticles (NP) as previously reported $(20,24)$. For in vitro transfection, NP was mixed with 100 pmol siRNA or $2 \mu \mathrm{g}$ shRNA pDNA in the presence of $50 \mathrm{mM} \mathrm{NaCl}$ solution at a charge ratio (+/-) of 3/1. The NP and siRNA or shRNA pDNA complex (nanoplex) was kept at room temperature for 15 min. The nanoplex was diluted with culture medium containing $10 \%$ FBS and transfected into cells at a final concentration of $100 \mathrm{nM}$ siRNA or $2 \mu \mathrm{g} / \mathrm{ml}$ shRNA pDNA in the medium.

For in vivo transfection, NP was mixed with $10 \mu \mathrm{g}$ siRNA or shRNA pDNA in water at a charge ratio (+/-) of $1 / 1$. The nanoplex was kept at room temperature for $15 \mathrm{~min}$. Male $\mathrm{BALB} / \mathrm{c}$ nu/nu mice (6-8 weeks of age) were purchased from CLEA Japan Inc. (Tokyo, Japan). To generate KB tumor xenografts, $1 \times 10^{7}$ cells suspended in $50 \mu 1$ RPMI medium were inoculated subcutaneously into the mice. The tumor volume was calculated using the formula, tumor volume $=0.5 \mathrm{x} \mathrm{a} \mathrm{x} \mathrm{b}^{2}$, where $\mathrm{a}$ and $\mathrm{b}$ are the larger and smaller diameters, respectively. When the average volume of KB xenograft tumors reached about $100 \mathrm{~mm}^{3}$, the nanoplex of siRNA or shRNA pDNA was directly injected into xenografts.

Antiproliferative activity. KB, A549 and HeLa cells were seeded in 96-well plates $24 \mathrm{~h}$ prior to transfection. Cells at $30 \%$ confluence in the wells were transfected with siRNA or shRNA pDNA by NP and then incubated for $72 \mathrm{~h}$. In combined treatment of siRNA or shRNA pDNA with paclitaxel (PTX, Wako, Osaka, Japan), the cells were incubated for $48 \mathrm{~h}$ after transfection of siRNA or shRNA pDNA, and then treated with various concentrations of PTX for another $48 \mathrm{~h}$. Cell viability (\%) was measured by the WST-8 assay (Dojindo Laboratories, Kumamoto, Japan) as previously reported (25).

Western blot analysis. KB, A549 and HeLa cells were seeded in a $35-\mathrm{mm}$ culture dish $24 \mathrm{~h}$ before transfection. The cells were transfected with HER-2 siRNA or HER-2 shRNA pDNA, and then incubated for $48 \mathrm{~h}$. Cell protein extracts were prepared with sampling buffer containing 1\% Triton X-100 in phosphate-buffered saline (PBS), $\mathrm{pH}$ 7.4. After they were centrifuged at $10,000 \mathrm{x}$ g for $10 \mathrm{~min}$, the protein concentration of the supernatant was quantitated with the bicinchonic acid protein assay reagent (Pierce, Rockford, IL, USA). For the detection of $B$-actin protein, $10 \mu \mathrm{g}$ protein was separated by $12.5 \%$ SDS-PAGE, and for the detection of HER-2 protein, 10 $\mu \mathrm{g}$ protein was separated by $7.5 \%$ SDS-PAGE. They were then transferred to a polyvinylidene difluoride (PVDF) membrane (FluoroTrans ${ }^{\circledR}$ W, PALL Gelman Laboratory, Ann Arbor, MI, USA). Membranes were blocked in PBS containing $0.1 \%$ Tween-20 with $5 \%$ skimmed milk at $37^{\circ} \mathrm{C}$ for $1 \mathrm{~h}$. The blot of HER-2 protein was probed with rabbit anti-human HER-2 antibody (Lab Vision, Fremont, CA, USA). Goat anti-rabbit IgG peroxidase conjugate (Santa Cruz Biotechnology, Inc., Santa Cruz, CA, USA) was used as a secondary antibody. Blots of $B$-actin protein were probed with a mouse anti-human $\beta$-actin IgG peroxidase conjugate [ß-actin (C4) HRP, Santa Cruz Biotechnology, Inc.]. Immunoblots were detected using a SuperSignal West Pico Chemiluminescent Substrate (Pierce).

Caspase 3/7 activities. $\mathrm{KB}$ and A549 cells were seeded in a 35-mm culture dish and incubated overnight. Cells at $30 \%$ confluence in the wells were transfected with siRNA or shRNA pDNA by NP for $24 \mathrm{~h}$. For measuring caspase 3/7 activity, a homogeneous assay (Caspase-Glo ${ }^{\mathrm{TM}}$ 3/7 assay, Promega, Madison, WI, USA) was performed as previously reported (25).

In vivo therapy. In gene therapy by transfection of HER-2 siRNA or HER-2 shRNA pDNA alone, the nanoplexes of $10 \mu \mathrm{g}$ siRNA or $10 \mu \mathrm{g}$ shRNA pDNA per tumor were directly injected into xenografts on days 0,2 and 4 . In the combined therapy of trastuzumab with PTX, trastuzumab (Herceptin, Chugai Pharmaceutical Co., Ltd., Tokyo, Japan) at a dose of $20 \mathrm{mg} / \mathrm{kg}$ and PTX at a dose of $5 \mathrm{mg} / \mathrm{kg}$ were simultaneously injected i.p. and i.v., respectively, on day $0,3,6$ and 9. In the combined therapy of HER-2 siRNA or HER-2 shRNA pDNA with PTX, nanoplexes of $10 \mu \mathrm{g}$ siRNA or $10 \mu \mathrm{g}$ shRNA pDNA per tumor were directly injected into xenografts on days 0 , 3,6 and 9, and PTX at a dose of $5 \mathrm{mg} / \mathrm{kg}$ was injected i.v. on day 1, 4, 7 and 10. Tumor volume and mean survival days were measured. The data are shown as the mean \pm SD. Animal experiments were conducted with ethics approval from our institutional animal care and use committee.

Statistical analysis. Statistical differences between different groups were analyzed with one-way analysis of variance on ranks with Tukey-Kramer's post-hoc test. A $\mathrm{p} \leq 0.05$ was considered significant. For the animal study, statistical comparison was performed by Student's t-test. 
A

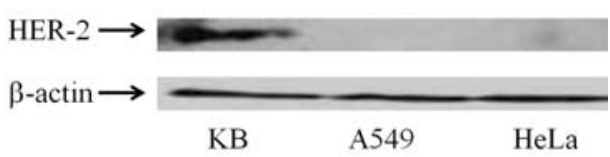

B

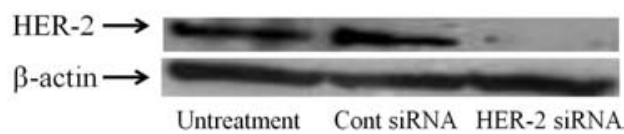

C

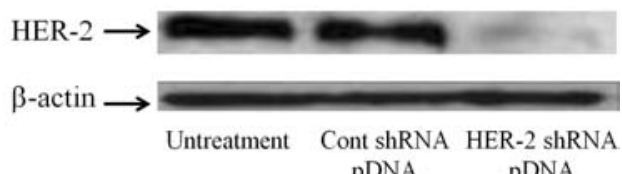

Figure 1. HER-2 expression in KB, A549 and HeLa cells (A) and suppression of HER-2 expression by transfection of HER-2 siRNA (B) or HER-2 shRNA pDNA (C) in KB cells. Expression of HER-2 protein was detected by Western blot analysis $48 \mathrm{~h}$ after transfection.

\section{Results}

Suppression of HER-2 protein by gene knockdown. HER-2 protein is a well-known protein overexpressed in many tumors and is related to apoptosis and cell growth (1). First, we investigated the expression of HER-2 mRNA in KB, A549 and HeLa cells by Western blot analysis (Fig. 1A). HER-2 protein was strongly expressed in KB cells, but not detected in A549 and HeLa cells; therefore, in subsequent experiments, we used KB cells as a HER-2 positive cell line, and A549 and HeLa cells as HER-2 negative cell lines.

Next, we confirmed the decreased expression of HER-2 protein by transfecting HER-2 siRNA or HER-2 shRNA pDNA into KB cells. In this study, we used lipid-based nanoparticles (NP) for siRNA and shRNA pDNA transfection as previously reported $(20,24,26)$. When transfected into KB cells, HER-2 siRNA or HER-2 shRNA pDNA strongly inhibited the expression of HER-2 protein, but did not affect the expression of $\beta$-actin (Fig. 1B and C). Control constructs, Cont siRNA and Cont shRNA pDNA, did not affect the expression either HER-2 or $\beta$-actin protein in the cells.

Antiproliferative activity. We examined cell viability by WST-8 assay $48 \mathrm{~h}$ after transfection of HER-2 siRNA and HER-2 shRNA pDNA into KB, A549 and HeLa cells. In the transfection of HER-2 siRNA and HER-2 shRNA pDNA, a decrease of cell viability was significantly observed in KB cells, but not in A549 and HeLa cells (Fig. 2A and B). In contrast, Cont siRNA and Cont shRNA pDNA did not affect cell viability in KB, A549 and HeLa cells.

Next, to examine the effect of HER-2 suppression on the activity of apoptosis-associated enzymes, we measured caspase $3 / 7$ activity $48 \mathrm{~h}$ after transfection with HER-2 siRNA or HER-2 shRNA pDNA (Fig. 2C and D). Transfection of HER-2 siRNA and HER-2 shRNA pDNA in KB cells increased caspase 3/7 activities about 1.4- and 1.5-fold higher than those of Cont siRNA and Cont shRNA pDNA,
A

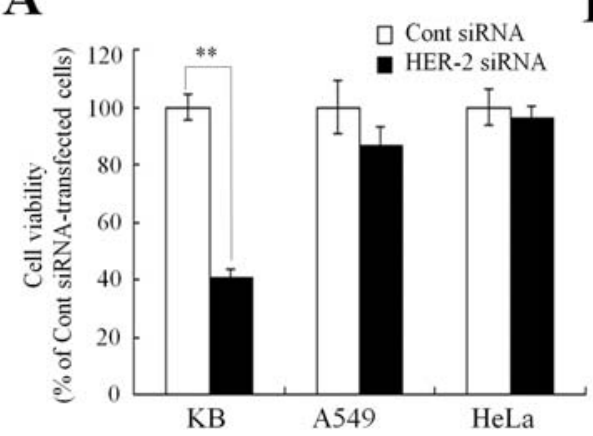

C

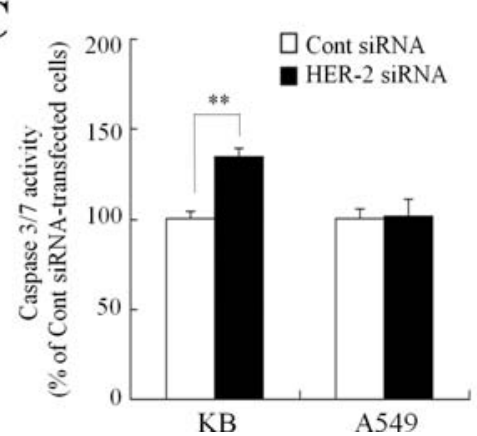

B

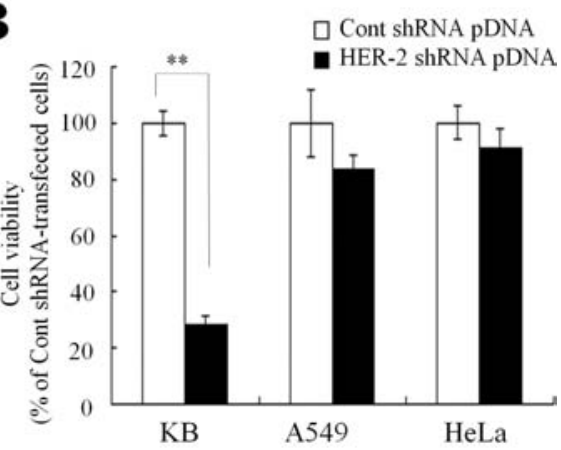

D

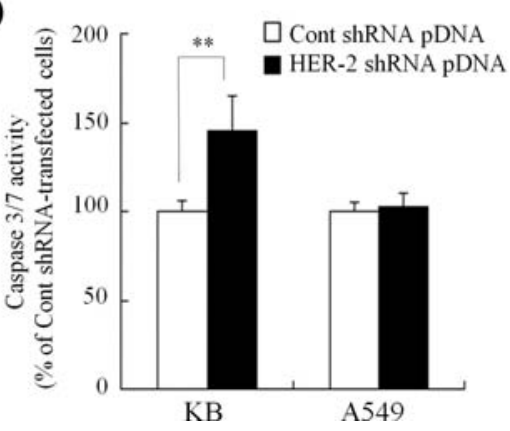

Figure 2. Antiproliferative and caspase $3 / 7$ activities $48 \mathrm{~h}$ after transfection of HER-2 siRNA or HER-2 shRNA pDNA into cells. After transfection with HER-2 siRNA or HER-2 shRNA pDNA, cell viability in KB, A549 and HeLa cells (A and B) and caspase 3/7 activity in KB and A549 cells (C and D) were measured. Each column shows the mean $\pm \mathrm{SD}(\mathrm{n}=3) .{ }^{* *} \mathrm{P}<0.01$, compared with Cont siRNA in A and $\mathrm{C}$, and with Cont shRNA pDNA in $\mathrm{B}$ and $\mathrm{D}$. 
A

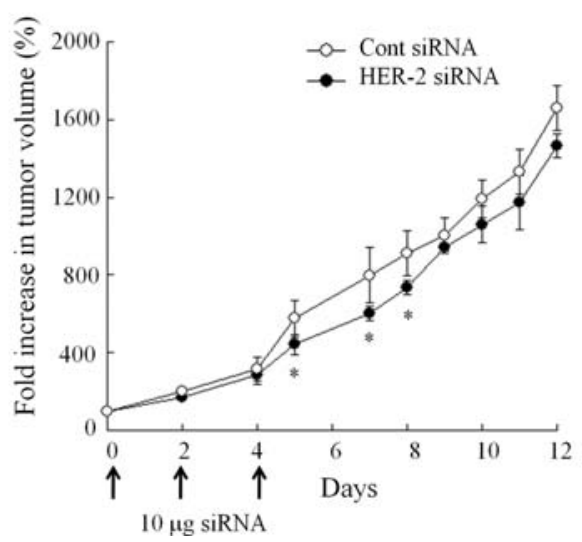

B

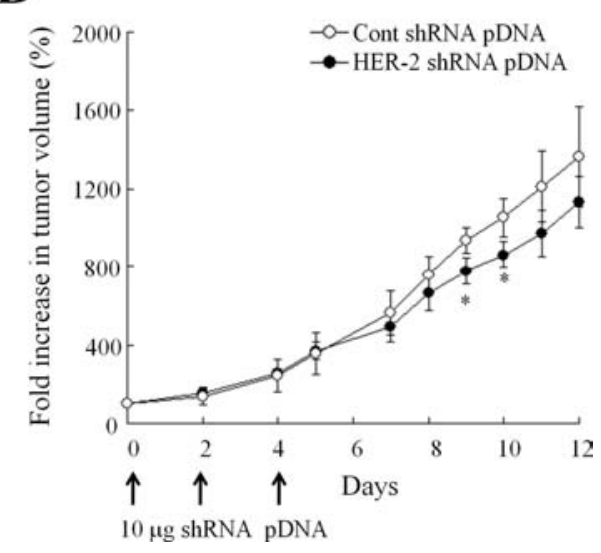

Figure 3. In vivo gene therapy of KB tumor xenografts. siRNA (A) or shRNA pDNA (B) was injected directly into the tumor three times (day 0,2 and 4). The results are the mean $\pm \mathrm{SE}(\mathrm{n}=4-6)$. ${ }^{*} \mathrm{P}<0.05$, compared with Cont siRNA in A and with Cont shRNA pDNA in $\mathrm{B}$.
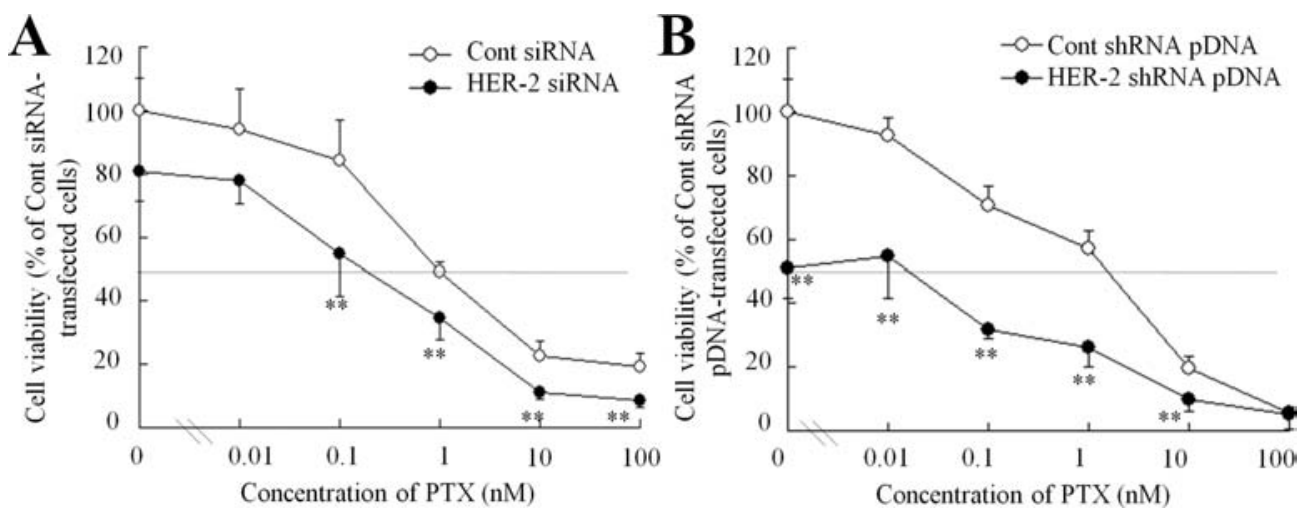

Figure 4. Dose effect of PTX on cytotoxicity in KB cells in the presence of siRNA (A) or shRNA pDNA (B). KB cells were transfected for $48 \mathrm{~h}$ with siRNA or shRNA pDNA. The cells were treated with various concentrations of PTX and incubated for another $48 \mathrm{~h}$. The number of viable cells was determined by WST-8 assay, $\mathrm{n}=4$ for each sample. ${ }^{*} \mathrm{P}<0.05$ and ${ }^{* *} \mathrm{P}<0.01$, compared with Cont siRNA in A and with Cont shRNA pDNA in $\mathrm{B}$.

respectively $(\mathrm{p}<0.01)$, whereas did not increase activity in A549 cells. These results suggested that HER-2 siRNA and HER-2 shRNA pDNA induced the inhibition of cell growth via apoptosis in HER-2-positive cells.

In vivo gene therapy in $K B$ tumor xenografts. We evaluated the antitumor effect by direct injection into KB tumor xenografts with the nanoplex of HER-2 siRNA or HER-2 shRNA pDNA. In vivo transfections of siRNA and shRNA pDNA were performed three times as previously reported (20). In mice treated with HER-2 siRNA, the growth of KB tumors was significantly inhibited on day 5, 7 and 8 compared with mice treated with Cont siRNA (Fig. 3A). In mice treated with HER-2 shRNA pDNA, growth was significantly inhibited on day 9 and 10 compared with mice treated with Cont shRNA pDNA (Fig. 3B). The time of tumor suppression by HER-2 shRNA pDNA seemed to be delayed more than that of HER-2 siRNA. However, sustained suppression of tumor growth was not observed in the xenografts after injection of HER-2 siRNA and HER-2 shRNA pDNA. HER-2 activates cell survival pathways, which represents an advantage for tumor cells as they became resistant to chemotherapy-induced apoptosis (8). Down-regulation of HER-2 expression in tumor cells via activation of the apoptosis pathway will enhance cytotoxicity by chemotherapy; therefore, for complete tumor regression, combination therapy with HER-2 siRNA and chemotherapy was examined.

In vitro combination therapy with PTX. It has been reported that the HER-2 antibody trastuzumab enhances the antitumor activity of PTX against HER-2-overexpressing human breast cancer xenografts (27), and combination therapy with trastuzumab and PTX has been useful for the clinical treatment of breast cancer (21-23). Therefore, we evaluated the in vitro growth inhibitory effect by the combination of HER-2 siRNA or HER-2 shRNA pDNA with PTX. Forty-eight hours after the transfection of siRNA or shRNA pDNA into KB cells, the cells was treated with PTX for another $48 \mathrm{~h}$. Cells transfected with HER-2 siRNA showed 3.2-fold higher sensitivity to PTX $\left(\mathrm{IC}_{50}=0.3 \mathrm{nM}\right)$ than those transfected with Cont 
A

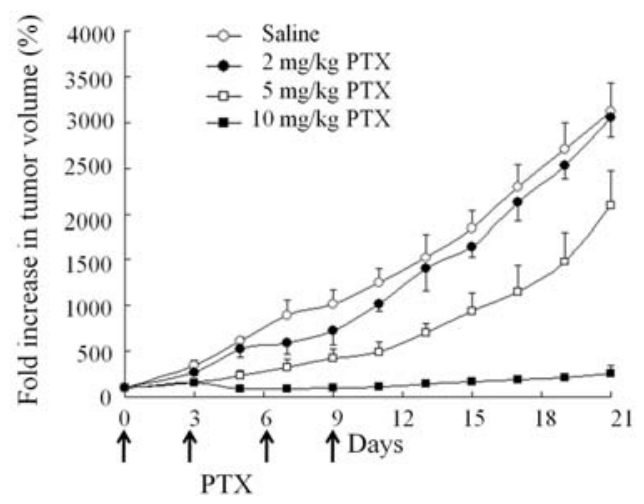

B

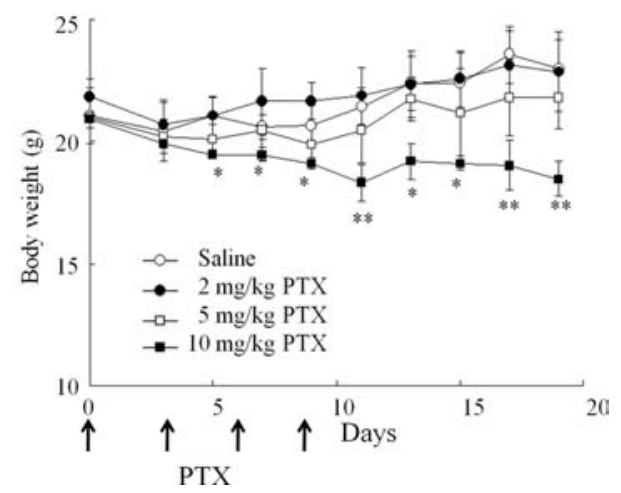

Figure 5. Dose-dependent effect of PTX on antitumor effect (A) and body weight change (B) for KB tumor xenografts. PTX was injected i.v. at a dose of 2, 5 and $10 \mathrm{mg} / \mathrm{kg}$ on days $0,3,6$ and 9 . Tumor volume $(\mathrm{A})$ and body weight change (B) were measured after starting treatment. Data are the mean $\pm \mathrm{SE}$, $\mathrm{n}=3$ for each group. ${ }^{*} \mathrm{P}<0.05$ and ${ }^{* *} \mathrm{P}<0.01$; compared with mice injected with saline.

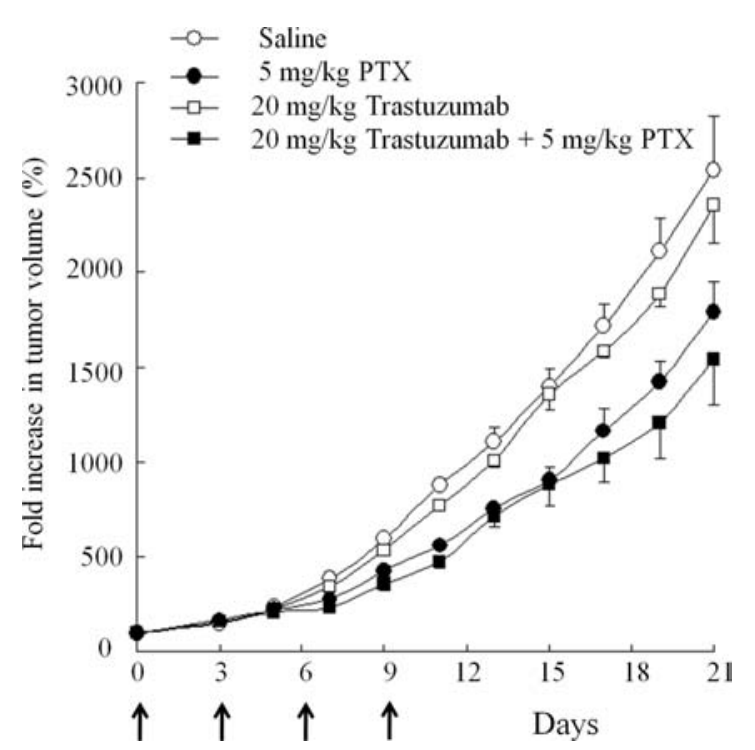

Figure 6. In vivo combination therapy of trastuzumab and PTX for KB tumor xenografts. When the average volume of KB tumor xenografts reached $100 \mathrm{~mm}^{3}$ (day 0), trastuzumab at $20 \mathrm{mg} / \mathrm{kg}$ (i.p.) and/or PTX (i.v.) at $5 \mathrm{mg} / \mathrm{kg}$ were injected on days $0,3,6$ and $9 . \mathrm{n}=3$ for each group.

siRNA $\left(\mathrm{IC}_{50}=0.97 \mathrm{nM}\right)$ (Fig. 4A). Cells transfected with HER-2 shRNA pDNA showed 92.5-fold higher sensitivity to PTX $\left(\mathrm{IC}_{50}=0.03 \mathrm{nM}\right)$ than those with Cont shRNA pDNA $\left(\mathrm{IC}_{50}=2.7 \mathrm{nM}\right)$ (Fig. 4B). These data indicated that suppression of HER-2 expression may increase sensitivity to PTX.

Combined therapy for KB tumor xenografts. To determine the optimal dose of PTX to evaluate combination therapy in vivo, PTX was administered at doses of 2, 5 and $10 \mathrm{mg} / \mathrm{kg}, 4$ times at three-day intervals. The tumor suppressive effect by PTX was dose-dependent; $5 \mathrm{mg} / \mathrm{kg}$ PTX inhibited the tumor growth of KB xenografts moderately but not at $2 \mathrm{mg} / \mathrm{kg}$ (Fig. 5A). There were no significant differences in mouse body weight changes after PTX administration at doses of 2 and $5 \mathrm{mg} / \mathrm{kg}$; however, body weight at $10 \mathrm{mg} / \mathrm{kg}$ PTX significantly decreased as a side effect (Fig. 5B); therefore, we decided to use $5 \mathrm{mg} / \mathrm{kg}$ PTX for the following combination therapy.

First, we investigated whether HER-2 inhibition by trastuzumab enhanced the antitumor activity of PTX for KB tumor xenografts. Trastuzumab was simultaneously i.p. injected at a dose of $20 \mathrm{mg} / \mathrm{kg}$, as previously reported (28), on days $0,3,6$ and 9 along with PTX; however, trastuzumab treatment alone could not inhibit tumor growth, and the combination with PTX could not significantly enhance the antitumor effect by PTX (Fig. 6).

Next, we evaluated the efficacy of combination therapy of HER-2 siRNA or HER-2 shRNA pDNA plus PTX in inhibiting the growth of KB tumors. Nanoplexes of $10 \mu \mathrm{g}$ HER-2 siRNA or HER-2 shRNA pDNA per tumor were directly injected into xenografts four times (days 0, 3, 6 and 9). PTX was intravenously administered at a dose of $5 \mathrm{mg} / \mathrm{kg} 24 \mathrm{~h}$ after the injections of nanoplexes (days 1, 4, 7 and 10). HER-2 siRNA or HER-2 shRNA pDNA treatment alone could not suppress tumor growth, but HER-2 siRNA and HER-2 shRNA pDNA with PTX treatment significantly suppressed tumor growth on day 7, 9 and 11, and day 9, 11 and 13, respectively, compared with PTX treatment alone (Figs. 7A and 8A). The median survival time with HER-2 siRNA combined treatment (31.5 days) was slightly longer than with HER-2 siRNA (28.5 days) or PTX (30.0 days) alone (Fig. 7B). The median survival time with HER-2 shRNA pDNA combined treatment (42.5 days) largely extended than that of the HER-2 shRNA pDNA (32.0 days) or PTX (35.0 days) alone (Fig. 8B). Transfection with siRNA, shRNA pDNA, PTX injection $(5 \mathrm{mg} / \mathrm{kg}$ ) alone, or their combinations did not alter the change in body weight during 3 weeks of treatment (data not shown). These data suggested that combination therapy of PTX with HER-2 siRNA or HER-2 shRNA pDNA was more effective than that of PTX with trastuzumab.

\section{Discussion}

Previously, we reported that NP-delivered HER-2 siRNA suppressed HER-2 expression, but insufficiently inhibited KB tumor growth (20). In this study, we investigated the anti- 


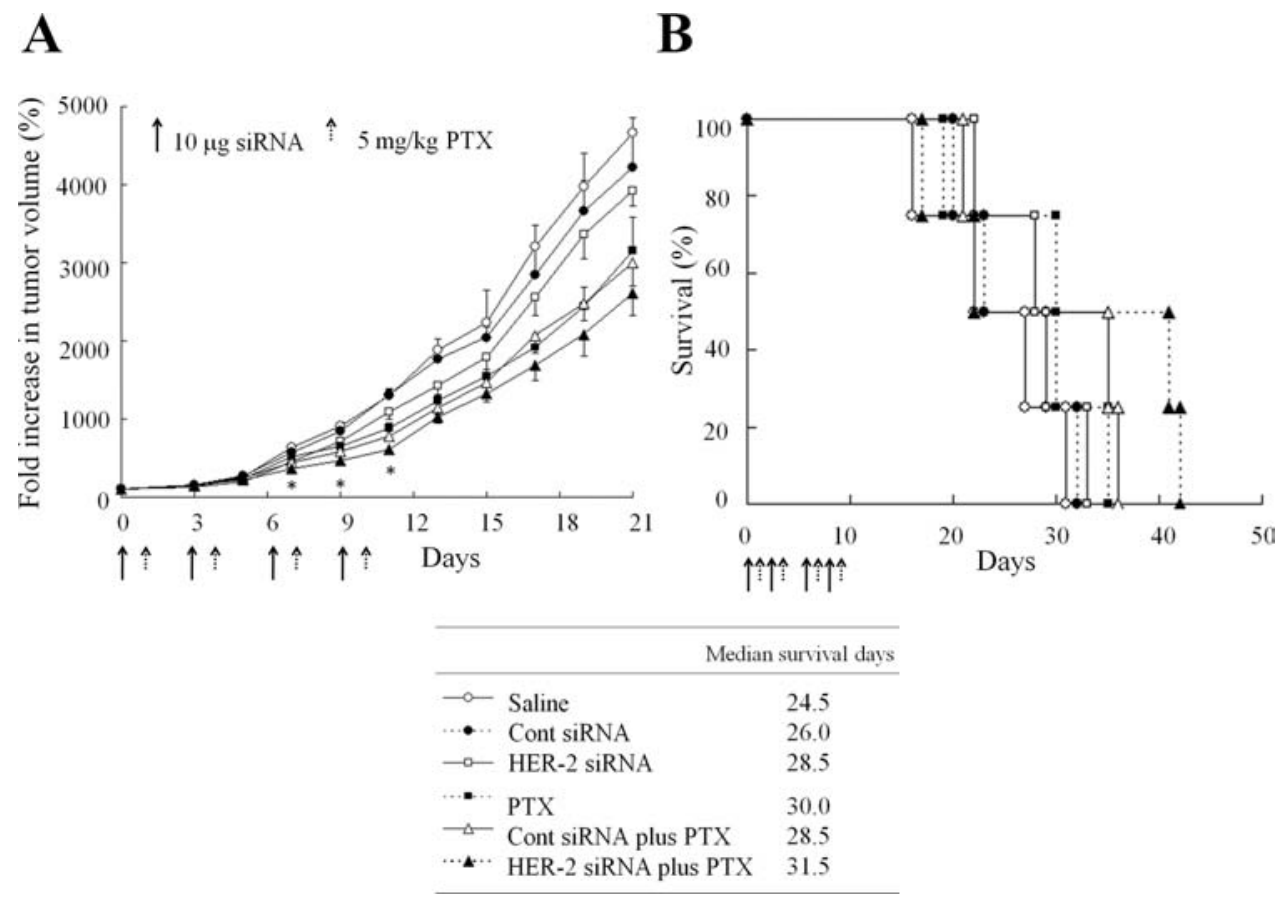

Figure 7. In vivo combination therapy of HER-2 siRNA and PTX for KB tumor xenografts. When the average volume of KB tumor xenografts reached $100 \mathrm{~mm}^{3}$ (day 0), HER-2 or Cont siRNA were directly injected into the tumor four times (days $0,3,6$ and 9). PTX at a dose of $5 \mathrm{mg} / \mathrm{kg}$ was injected i.v. four times (days 1, 4, 7 and 10). Tumor volume (A) and mean survival days (B) were measured after starting treatment. In A, data are shown as the mean \pm SE. $n=3$ for each group. ${ }^{*} \mathrm{P}<0.05$; compared with mice injected with PTX.

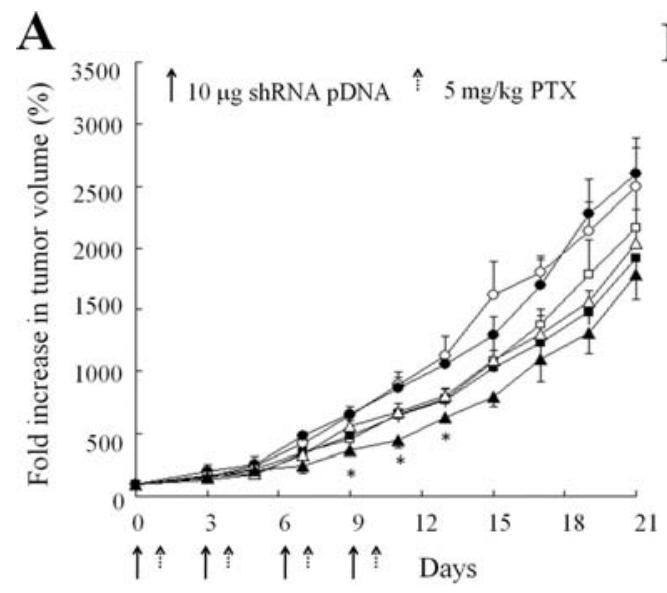

B

\begin{tabular}{lc} 
& Median survival days \\
\hline$\multimap-$ Saline & 28.5 \\
$\ldots$ Cont shRNA pDNA & 27.0 \\
$\because-$ HER-2 shRNA pDNA & 32.0 \\
$\ldots$ PTX & 35.0 \\
$\rightarrow$ Cont shRNA pDNA plus PTX & 30.5 \\
$\ldots$ - HER-2 shRNA pDNA plus PTX & 42.5 \\
\hline
\end{tabular}

Figure 8. In vivo combination therapy with HER-2 shRNA pDNA and PTX for KB tumor xenografts. Experimental conditions were the same as in Fig. 7.

tumor effect of NP transfection of HER-2 shRNA pDNA compared with HER-2 siRNA into KB cells and tumor xenografts. Furthermore, we examined their combination therapy with PTX.

The intracellular localization of HER-2 siRNA and HER-2 shRNA pDNA after transfection is crucial for its successful function. A difference in time of the antitumor effect between
HER-2 siRNA and HER-2 shRNA pDNA was slightly observed (Fig. 3). In combination therapy with PTX, HER-2 shRNA pDNA exhibited marked extension of mean survival days compared with HER-2 siRNA (Figs. 7B and 8B). HER-2 siRNA can quickly trigger specific degradation of HER-2 mRNA after transfection into cytoplasm. On the other hand, HER-2 shRNA pDNA must be transferred into the nucleus 
for the HER-2 shRNA expression. It can therefore suppress the expression of HER-2 mRNA for a long period by sustained transcription of HER-2 shRNA from pDNA. This might be one of the reasons why the combination of HER-2 shRNA pDNA and PTX treatment extended the mean survival days.

Trastuzumab is widely used as a standard therapy for patients with HER-2-overexpressing metastatic breast cancer with clear evidence of clinical efficacy as a single agent or in combination with chemotherapeutic agents (29). It has also been reported that trastuzumab enhances the antitumor activity of PTX and doxorubicin against HER-2-overexpressing human breast cancer xenografts (27); however, in our results, trastuzumab was not effective for KB culture cells (data not shown) and KB tumor xenografts as a single agent. Moreover, trastuzumab did not increase the sensitivity in combination with PTX (Fig. 6) like HER-2 siRNA and HER-2 shRNA pDNA pretreatment (Figs. 7A and 8A). This finding correspond well with the result that $60-70 \%$ metastatic patients with HER-2-positive tumors appear intrinsically resistant to trastuzumab as a sole therapy $(30,31)$. Furthermore, trastuzumab alone or in combination with docetaxel or PTX was not effective in clinical trials for HER-2-positive prostate tumors (32,33). A cell line developed from a patient resistant to trastuzumab treatment was found to maintain high levels of HER-2 expression without identifiable mutations (34). These findings might explain why trastuzumab was not effective for KB tumor xenografts. In this study, combination therapy with HER-2 siRNA or HER-2 shRNA pDNA increased the inhibitory effect of PTX and mean survival days in KB tumor xenografts, suggesting that HER-2 RNAi therapy might be effective for trastuzumab-resistant cells in combination with chemotherapy.

In our study, the mechanism of the enhanced antitumor effect for chemotherapy by HER-2 suppression remains unclear. We speculated that HER-2 suppression contributed to the reduction of antiapoptotic signaling and then increased the sensitivity to chemotherapy. As another mechanism, PTX increased the liposome-delivered transfection efficiency by inhibiting targeting endosomes to lysosomes $(35,36)$. We confirmed that PTX treatment increased 2-fold in gene expression by NP in KB cells (data not shown). Therefore, using the combined lipid-mediated transfection of genes with PTX might be a powerful technique due to the effect of enhanced gene therapy. Combination therapy has the potential to reduce the side effects of PTX.

In conclusion, we demonstrated the potential of HER-2 siRNA and HER-2 shRNA pDNA to increase the sensitivity of PTX in HER-2-positive KB cells and tumor xenografts using NP delivery. Suppression of HER-2 expression significantly inhibited cell growth and induced caspase $3 / 7$ activity in KB cells. Thus, the combination of HER-2 siRNA and PTX may serve as a novel tool for gene therapy with fewer adverse effects.

\section{Acknowledgements}

We thank Ms. Mako Kato for assistance with the experimental study. This project was supported in part by a Grant-in-Aid for Scientific Research from the Ministry of Education, Culture, Sports, Science, and Technology of Japan, and by the Open Research Center Project.

\section{References}

1. Bacus SS, Zelnick CR, Plowman G and Yarden Y: Expression of the erbB-2 family of growth factor receptors and their ligands in breast cancers. Implication for tumor biology and clinical behavior. Am J Clin Pathol 102: S13-S24, 1994.

2. McCann A, Johnston PA, Dervan PA, Gullick WJ and Carney DN: c-erB-2 oncoprotein expression in malignant and nonmalignant breast tissue. Ir J Med Sci 158: 137-140, 1989.

3. Sasaki N, Kudoh K, Kita T, Tsuda H, Furuya K and Kikuchi Y: Effect of HER-2/neu overexpression on chemoresistance and prognosis in ovarian carcinoma. J Obstet Gynaecol Res 33: 17-23, 2007.

4 Hirsch FR, Franklin WA, Veve R, Varella-Garcia M and Bunn PA Jr: HER2/neu expression in malignant lung tumors. Semin Oncol 29: 51-58, 2002

5. Giri DK, Wadhwa SN, Upadhaya SN and Talwar GP: Expression of NEU/HER-2 oncoprotein (p185neu) in prostate tumors: an immunohistochemical study. Prostate 23: 329-336, 1993.

6. Mondi MM, Rich R, Ituarte P, Wong M, Bergman S, Clark $\mathrm{OH}$ and Perrier ND: HER2 expression in thyroid tumors. Am Surg 69: 1100-1103, 2003.

7. Tomaszewska R, Okon K, Nowak K and Stachura J: HER-2/ $\mathrm{Neu}$ expression as a progression marker in pancreatic intraepithelial neoplasia. Pol J Pathol 49: 83-92, 1998.

8. Zheng L, Ren JQ, Li H, Kong ZL and Zhu HG: Downregulation of wild-type $\mathrm{p} 53$ protein by HER-2/neu mediated PI3K pathway activation in human breast cancer cells: its effect on cell proliferation and implication for therapy. Cell Res 14: 497-506, 2004.

9. Witters LM, Santala SM, Engle L, Chinchilli V and Lipton A: Decreased response to paclitaxel versus docetaxel in HER-2/ neu transfected human breast cancer cells. Am J Clin Oncol 26: 505-514, 2003.

10. Suzuki T, Anderegg B, Ohkawa T, Irie A, Engebraaten O, Halks-Miller M, Holm PS, Curiel DT, Kashani-Sabet M and Scanlon KJ: Adenovirus-mediated ribozyme targeting of HER-2/ neu inhibits in vivo growth of breast cancer cells. Gene Ther 7: 241-248, 2000

11. Bi F, Fan D, Hui H, Wang C and Zhang X: Reversion of the malignant phenotype of gastric cancer cell SGC7901 by c-erbB-2specific hammerhead ribozyme. Cancer Gene Ther 8: 835-842, 2001.

12. Lui VW, He Y and Huang L: Specific down-regulation of HER2/neu mediated by a chimeric U6 hammerhead ribozyme results in growth inhibition of human ovarian carcinoma. Mol Ther 3: 169-177, 2001.

13. Rait AS, Pirollo KF, Ulick D, Cullen K and Chang EH: HER-2targeted antisense oligonucleotide results in sensitization of head and neck cancer cells to chemotherapeutic agents. Ann N Y Acad Sci 1002: 78-89, 2003

14. Rait AS, Pirollo KF, Xiang L, Ulick D and Chang EH: Tumortargeting, systemically delivered antisense HER-2 chemosensitizes human breast cancer xenografts irrespective of HER-2 levels. Mol Med 8: 475-486, 2002.

15. Shen M, Feng Y, Ge B, Wu Z and Zhu M: Liposome-C-erbB2 antisense oligodoxynucleotides in human ovarian cancer cells. Chin Med J 114: 735-737, 2001.

16. Pirollo KF, Rait A, Zhou Q, Hwang SH, Dagata JA, Zon G, Hogrefe R,I Palchik G and Chang EH: Materializing the potential of small interfering RNA via a tumor-targeting nanodelivery system. Cancer Res 67: 2938-2943, 2007.

17. Tan WB, Jiang S and Zhang Y: Quantum-dot based nanoparticles for targeted silencing of HER2/neu gene via RNA interference. Biomaterials 28: 1565-1571, 2007.

18. Ren XL, Xu YM, Bao W, Fu HJ, Wu CG, Zhao Y, Li ZK, Zhang J, Li SQ, Chen WQ, Wang T, Zhang R, Zhang LH, Qian GS, Chen SY, Jia LT and Yang AG: Inhibition of nonsmall cell lung cancer cell proliferation and tumor growth by vector-based small interfering RNAs targeting HER2/neu. Cancer Lett 281: 134-143, 2009.

19. Hogrefe RI, Lebedev AV, Zon G, Pirollo KF, Rait A, Zhou Q, $\mathrm{Yu}$ W and Chang EH: Chemically modified short interfering hybrids (siHYBRIDS): nanoimmunoliposome delivery in vitro and in vivo for RNAi of HER-2. Nucleosides Nucleotides Nucleic Acids 25: 889-907, 2006.

20. Yoshizawa T, Hattori Y, Hakoshima M, Koga K and Maitani Y: Folate-linked lipid-based nanoparticles for synthetic siRNA delivery in KB tumor xenografts. Eur J Pharm Biopharm70: 718-725, 2008. 
21. Okawa Y, Sugiyama K, Aiba K, Hirano A, Uno S, Hagino T, Kawase K, Shioya H, Yoshida K, Usui N, Kobayashi M and Kobayashi T: Successful combination therapy with trastuzumab and Paclitaxel for adriamycin- and docetaxel-resistant inflammatory breast cancer. Breast Cancer 11: 309-312, 2004.

22. Hayashi H, Kimura M, Yoshimoto N, Tsuzuki M, Tsunoda N, Fujita T, Yamashita T and Iwata H: A case of HER2-positive male breast cancer with lung metastases showing a good response to trastuzumab and paclitaxel treatment. Breast Cancer 16 136-140, 2009.

23. Bullock K and Blackwell K: Clinical efficacy of taxane-trastuzumab combination regimens for HER-2-positive metastatic breast cancer. Oncologist 13: 515-525, 2008.

24. Hattori Y, Ding WX and Maitani Y: Highly efficient cationic hydroxyethylated cholesterol-based nanoparticle-mediated gene transfer in vivo and in vitro in prostate carcinoma PC-3 cells. J Control Release 120: 122-130, 2007.

25. Hattori Y, Fukushima M and Maitani Y: Non-viral delivery of the connexin 43 gene with histone deacetylase inhibitor to human nasopharyngeal tumor cells enhances gene expression and inhibits in vivo tumor growth. Int J Oncol 30: 1427-1439, 2007.

26. Hattori Y, Yoshizawa T, Koga $\mathrm{K}$ and Maitani Y: $\mathrm{NaCl}$ induced high cationic hydroxyethylated cholesterol-based nanoparticlemediated synthetic small interfering RNA transfer into prostate carcinoma PC-3 cells. Biol Pharm Bull 31: 2294-2301, 2008.

27. Baselga J, Norton L, Albanell J, Kim YM and Mendelsohn J: Recombinant humanized anti-HER2 antibody (Herceptin) enhances the antitumor activity of paclitaxel and doxorubicin against HER2/neu overexpressing human breast cancer xenografts. Cancer Res 58: 2825-2831, 1998.

28. Fujimoto-Ouchi K, Sekiguchi F, Yasuno H, Moriya Y, Mori K and Tanaka Y: Antitumor activity of trastuzumab in combination with chemotherapy in human gastric cancer xenograft models. Cancer Chemother Pharmacol 59: 795-805, 2007.
29. Hall PS and Cameron DA: Current perspective-trastuzumab. Eur J Cancer 45: 12-18, 2009.

30. Burris H III, Yardley D, Jones S, Houston G, Broome C, Thompson D, Greco FA, White M and Hainsworth J: Phase II trial of trastuzumab followed by weekly paclitaxel/carboplatin as first-line treatment for patients with metastatic breast cancer. J Clin Oncol 22: 1621-1629, 2004.

31. Vogel CL, Cobleigh MA, Tripathy D, Gutheil JC, Harris LN, Fehrenbacher L, Slamon DJ, Murphy M, Novotny WF, Burchmore M, Shak S, Stewart SJ and Press M: Efficacy and safety of trastuzumab as a single agent in first-line treatment of HER2-overexpressing metastatic breast cancer. J Clin Oncol 20: 719-726, 2002.

32. Morris MJ, Reuter VE, Kelly WK, Slovin SF, Kenneson K, Verbel D, Osman I and Scher HI: HER-2 profiling and targeting in prostate carcinoma. Cancer 94: 980-986, 2002.

33. Lara PN Jr, Chee KG, Longmate J, Ruel C, Meyers FJ, Gray CR, Edwards RG, Gumerlock PH, Twardowski P, Doroshow JH and Gandara DR: Trastuzumab plus docetaxel in HER-2/neu-positive prostate carcinoma: final results from the California Cancer Consortium Screening and Phase II Trial. Cancer 100: 2125-2131, 2004.

34. Tanner M, Kapanen AI, Junttila T, Raheem O, Grenman S, Elo J, Elenius $\mathrm{K}$ and Isola $\mathrm{J}$ : Characterization of a novel cell line established from a patient with Herceptin-resistant breast cancer. Mol Cancer Ther 3: 1585-1592, 2004.

35. Hasegawa S, Hirashima N and Nakanishi M: Microtubule involvement in the intracellular dynamics for gene transfection mediated by cationic liposomes. Gene Ther 8: 1669-1673, 2001.

36. Nair RR, Rodgers JR and Schwarz LA: Enhancement of transgene expression by combining glucocorticoids and anti-mitotic agents during transient transfection using DNA-cationic liposomes. Mol Ther 5: 455-462, 2002. 